\title{
Can Probabilistic Cognitive Modeling Explain Adoption Behavior of Smartphone Apps Gathering Private Data?
}

\author{
Tim Schürmann Jan Smirny Sina Zimmermann Joachim Vogt \\ Work and Engineering Psychology Group, Technische Universität Darmstadt \\ Corresponding Author: schuermann@psychologie.tu-darmstadt.de
}

\begin{abstract}
Predominant perspectives in privacy behavior research describe human decision making as boundedly rational, cognitively biased, and driven by heuristics. This description stands in contrast to Bayesian decision theory, which describes decision making as mathematically rational utility maximization. Recently, research has proposed rational process modeling as a way of considering justified criticisms of Bayesian models with regards to psychological plausibility. In rational process modeling, locally irrational behavior emerges from an approximation of Bayesian inference focused on the attribution of cognitive resources. In two online surveys, we have applied a rational process model to predict the choice behavior of 103 and 113 participants. They saw 3 (study 1) and 2 (study 2) app store offerings of fictional smartphone apps related to travel or messaging and decided whether to use them. Stated preferences for features of these apps were gathered and sampled from until triggering a probabilistic stopping rule. Then, they were integrated via Bayes' rule and used to predict service adoption. Depending on the number of samples, the model would show behavior ranging from extreme probability matching to near-deterministic utility maximization. We compared the model prediction of observed participant behavior to three alternative models by using Bayes Factors: a deterministic Bayesian model, the takethe-best heuristic, and a probabilistic variant of said heuristic. We found that the data give the most support to the proposed rational process model, although all models tended to overestimate service adoption. We connect our results to previous work in cognitive science and online privacy research and outline approaches derived from the model to encourage privacy-preserving user behavior through interventions.
\end{abstract}

\section{Introduction}

The extensive collection of data from contemporary online services allows for many user-friendly features, but also poses an immense threat to these users' privacy. Even though many users state their concern about the loss of privacy enabled by extensive data collection when probed, they tend to disclose personal data. This behavior has been termed Privacy Paradox [1]. Although it has received significant attention from psychology [2], economics [3], and related disciplines [4], no academic consensus has yet been reached regarding the origin of the phenomenon. Recent developments in the area of computational cognitive modeling however shine a new light on human decision making in general, and online privacy-related user behavior specifically. In this article, we will first outline the current state of research regarding the privacy paradox, and computational modeling of human choice behavior. Furthermore, we will describe two empirical studies that investigate the fit between the predictions of four cognitive models and user behavior.

\subsection{State of research}

Previous research has provided different answers as to why privacy disclosure behavior shows paradoxical tendencies [1], [5]-[8], and even whether it does at all [2], [4]. For example, [1] consider the Privacy Paradox to emerge from a gap between disclosure intentions and disclosure behavior. Contesting views distinguish between general attitudes and more situation-specific factors [6], and state that the Privacy Paradox constitutes a gap between attitudes and intentions rather than intentions and behavior. Some researchers assume these intentions to be derived from a somewhat rational deliberation concerning the perceived risks and benefits of a given choice [9]. This line of research can be considered an adaptation of expected utility theory [10]. A recent literature review [11] however finds that most explanatory accounts of the Privacy Paradox have in common that they challenge the assumption of rational decision making for online users, as it finds two lines of argument most prominently represented: that privacy-related decisions are affected by cognitive biases, and that human decision making can only be boundedly rational [12]. These cognitive biases are generally based on prospect theory [13] when referenced in online privacy research and refer to several observed deviations from mathematical rationality, for example distorted probability weighting and temporal discounting of rewards [14].

While the introduced lines of research differ in their explanation of privacy behavior, most have in common that they consider the decision process of disclosing private information to be subject to uncertainty. However, they do not offer a formal proposal on how uncertainty affects user decision making or how individual preference formation informs choice behavior. These lines of research can therefore mostly be classified 
as verbal-conceptual or mathematical models according to a classification by [15]. Models of human behavior that belong to these categories usually do not involve precise processlevel specifications, and therefore lack explicitness about which behavior is predicted [16]. We argue that this ambiguity involved in verbal theorizing about privacy-related decision making is partly responsible for the multiple theoretical explanations surrounding the prediction of user behavior. To our knowledge, none of the introduced lines of research claims that the specific privacy-related decision process differs from general, domain-free decision making. This enables us to employ formalized cognitive models of general decision making that involve precise predictions about the transition from preferences to choices and the management of uncertainty.

\subsection{Cognitive modeling}

A cognitive model attempts to predict a system's behavior by approximating the generative process driving it. A Bayesian cognitive model assumes that this process can be described as approximately following Bayes' rule, which is the mathematically rational way of integrating uncertain information. Following this line of argument, humans employ Bayes' rule when they integrate new information with their prior beliefs about the state of the world. They do so by assigning probabilities, or degrees of belief, to competing hypotheses. The posterior probability of a hypothesis upon seeing new evidence $P\left(h_{i} \mid d\right)$ is proportional to the likelihood of the evidence given the hypothesis $P\left(d \mid h_{i}\right)$ times the prior probability of the hypothesis $P\left(h_{i}\right)$. Equation 1 shows this process, repeats the step over all considered hypotheses and then normalizes in the denominator.

$$
P\left(h_{i} \mid d\right)=\frac{P\left(d \mid h_{i}\right) P\left(h_{i}\right)}{\sum_{h \in \mathcal{H}} P(d \mid h) P(h)}
$$

Bayesian decision theory [17] relates the concept of updating probabilities via Bayes' rule to decision making. When an agent tries to infer which option out of a set of options provides the most benefit, the agent ought to choose the one that maximizes expected utility, based on the subjective posterior probabilities attained using Bayes' rule. Because of the property of mathematical rationality adapted from Bayes' rule, Bayesian decision theory has been a benchmark against which the extent of human rationality has been measured for decades. Such investigations usually consider whether humans choose an option out of a set of options that maximizes the expected utility of potential outcomes. Inversely, the minimization of expected losses can also act as a decision criterion. Human behavior is then compared to the computationally optimal result of an ideal rational agent.
While references to these decision strategies have been made throughout the literature [9], [11], [14], [18], to our knowledge neither Bayesian nor non-Bayesian formalized cognitive models have been employed as explanations of privacy disclosure behavior. Meanwhile, computational psychology and cognitive science have developed and refined the formalization and comparison of cognitive models to investigate a wide array of phenomena involving human perception and decision making [16], [19]-[21]. In these domains, early research seemed to indicate that human cognition in general could not be described as Bayesian inference. For example, empirical evidence suggested that participants ignore prior knowledge [22], or that they weight probabilities in a distorted manner [13]. These results aligned with the assumption of bounded rationality in online privacy research that the human brain is not equipped to handle problems following Bayes' rule with real-world complexity, consequently relying on heuristics. Integrating over all possible hypotheses and deriving a posterior solution in a Bayesian manner might be possible for small, textbook examples, but on a realistic scale the hypothesis space would render solving a perception or decision problem computationally expensive.

However, these problems typically arise when considering Bayesian cognitive models from a purely computational perspective. [23] has outlined three levels of analysis on which information-processing systems can be investigated. While analysis on the computational level considers the goal and appropriateness of a computation, it explicitly makes no statement about the underlying processes and representations of the algorithmic level. Finally, the implementation level considers the biological realization of these processes in the human brain [24], but is beyond the scope of this article. In recent years, efforts have been made to develop cognitive models that consider both the computational and algorithmic levels of analysis and in doing so identify "psychologically plausible mechanisms that would allow the human mind to approximate optimal performance" [25]. These rational process models, shortened to RPM [26], typically rely on sampling from probability distributions instead of solving the computational problem directly. In doing so, they approach optimal performance the more samples they draw, until they eventually arrive at Bayesian performance given an infinite number of samples. Assuming knowledge about the world to be representable or describable as probability distributions, a sample from one of those distributions is analogous to one isolated instance of retrieving information from memory. In rational process models, sampling serves as a mathematical representation of this information retrieval. It is not, however, an observable process that participants go through consciously during an experiment, as for example in decision from experience paradigms [27]. [28] propose that by sampling, humans forego the need to represent or calculate full probability distributions, and that this process can explain 
several probabilistic reasoning errors such as base-rate neglect.

Two questions arise out of this line of argument: how many samples do humans draw to solve problems, and why would they settle for less than optimal performance? [29] suggest that human behavior seems to make use of only a very limited number of samples, because increasing that count does not reflect a sufficient decline in estimation errors. In a computational experiment, they found that for many scenarios concerning choices between two options, the optimal number of samples to draw is single-digit. It is rational for a samplingbased agent to invest more time in lowering their estimation error when the cost of drawing a sample decreases relative to the expected utility of a decision. The meta-level decision about the cognitive resources to invest in drawing more samples is made with the goal of utility maximization. This strategy, while globally rational, can produce behavior that appears locally irrational.

\subsection{Article outline}

As outlined above, a large portion of prior research on privacy-related decision making describes user behavior as boundedly rational and making use of heuristics. Therefore, it would seem at odds with Bayesian cognitive modeling, describing decision making as rational utility maximization. However, the establishment of rational process modeling provides the potential to resolve several findings that were initially interpreted as evidence against Bayesian cognitive modeling [28]: what appears to be distorted weighting of probabilities or the application of heuristic alternatives can now be investigated as locally irrational behavior that emerges from global rationality. This article applies the introduced RPM to a two-alternative forced-choice (2AFC) scenario in which participants were presented several online services and asked to choose between installing and not installing them. The services were presented as smartphone app offerings, advertising several features with and without ties to privacy-invasive functionality. Previous research has identified user behavior involving such functionality as seemingly paradoxical. While users state that they are concerned about loss of privacy, they decide to use potentially privacyinvasive services, therefore disclosing personal information. We argue that the foundation of users' choices is not well represented by asking for such relatively general privacy concerns, as many researchers have pointed out before us [11], [30], [31]. Instead, we assume that choice behavior relies on preferences sampled from an individual's choice history.

The present article compares the performance of the RPM to other decision-theoretical models that differ with regards to their computational properties. Section 2 will introduce these decision-theoretical models, alongside the applied evaluation methods and study design. Section 3 showcases descriptive and inferential results. Section 4 discusses implications regarding online privacy behavior and cognitive modeling of choice. In section 5, we derive possible starting points for privacy-enhancing interventions based on the user's choice process and lay out directions for future work.

\section{Study description and model formalization}

\subsection{Empirical studies}

103 participants (63 percent female, age $\mathrm{M}=31.5, \mathrm{SD}=11.8$ ) completed the first online survey after being recruited via clickworker.com, an online survey platform akin to Mechanical Turk that rewards participants financially for completing surveys. Out of 115 participants who started the survey, the aforementioned 103 completed it. To replicate the study results and generalize them through the introduction of apps from another usage category, we conducted an additional study. For the second online survey, we gathered 113 new participants (46 percent female, age $\mathrm{M}=29.8, \mathrm{SD}=5.7$ ), again via clickworker.com. In study 2 , one participant was excluded from analysis because their response pattern would have created only invalid feature comparisons in the models based on the take-the-best heuristic, leaving these models unable to arrive at a prediction. All remaining 112 participants of study 2 completed the survey. In both cases, no additional demographic requirements were set for participation.

Both studies were carried out in accordance with the recommendations of the ethics committee of Technische Universität Darmstadt with written informed consent from all participants. All participants gave written informed consent in accordance with the Declaration of Helsinki and were paid above German minimum wage criteria. The protocol was approved by the ethics committee of Technische Universität Darmstadt. After providing demographic information, participants were introduced to the service features relevant for the survey. The six features that were considered are shown in tables 1 and 2 .

Table 1.

Description and levels of service features for study 1 .

\begin{tabular}{cc}
\hline Feature & Description \\
\hline Price & Purchase price of the app (0.00€ / 2.99€) \\
Booking alert & Alert by the app at favorable booking time (yes / no) \\
Evaluation & Source of the hotel evaluation (customer / expert) \\
GPS & App access to GPS data (access / no access) \\
Calendar & App access to calendar (access / no access) \\
Discounts & Special discount when booking through app (yes / no) \\
\hline
\end{tabular}


Table 2 .

Description and levels of service features for study 2.

\begin{tabular}{|c|c|}
\hline Feature & Description \\
\hline Price & Purchase price of the app $(0.00 € / 2.99 €)$ \\
\hline User reviews & $\begin{array}{c}\text { User review score on a } 5 \text { star scale }(2.7 / 4.5 \\
\text { stars })\end{array}$ \\
\hline Encryption & End-to-end message encryption (yes / no) \\
\hline Multi-platform & $\begin{array}{c}\text { Availability on one or multiple major mobile op- } \\
\text { erating systems (yes / no) }\end{array}$ \\
\hline Contacts & App access to contacts (access / no access) \\
\hline Phone status & $\begin{array}{c}\text { App access to phone status and identity (access / } \\
\text { no access) }\end{array}$ \\
\hline
\end{tabular}

Separating the features into pairs of two, we asked the participants to rank the four cells of possible combinations for each pair according to their perceived desirability. The marginalized number of times a feature level was preferred over its alternative resulted in the odds of adopting a service given a present feature level. Converting these odds to probabilities resulted in the desirability of individual features. After providing the stated preference rankings, participants saw three consecutive, fictional offerings for online services designed to be reminiscent of app store offerings from common smart phone operating systems in the first survey, while participants in the second survey saw two others. Figure 1 showcases an example of these stimuli used translated to English. Specifics of the presentation remained unchanged between the two surveys. The app design was purposefully altered to limit the amount of information available to the features we considered. The apps in question differed in their distribution of available features but were all sub-sets of the feature list in tables 1 and 2. Contextually, the apps represented travel booking services for the first survey, and messenger services for the second one. Participants decided whether they wanted to install a single app at a time and were repeatedly instructed to treat stimuli as independent. This was implemented to avoid possible confusion over whether participants should decide between apps, instead of considering each app independently.

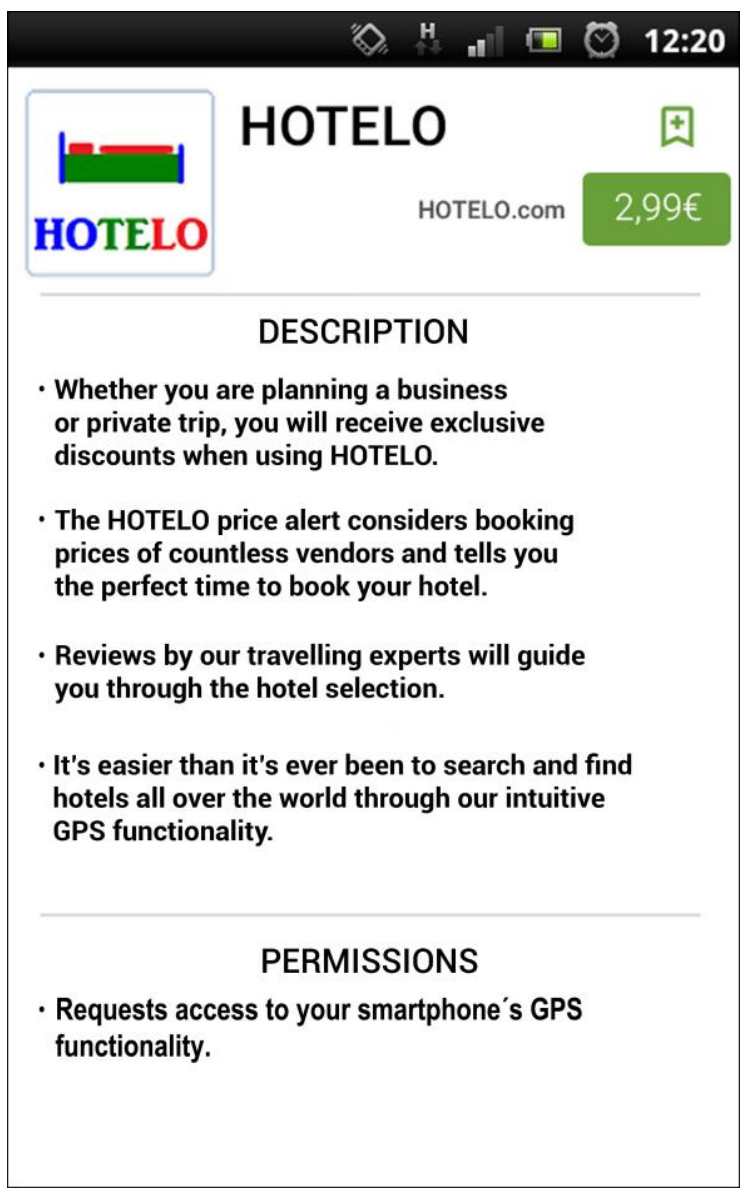

Figure 1. Stimulus for the first app of study 1.

\subsection{Model description}

In this section, we introduce three competing cognitive models next to the already introduced RPM and argue how they differ theoretically and computationally. Additionally, we showcase how each model processes individual feature preferences and arrives at a choice prediction regarding app installation.

\subsubsection{Rational Process Model (RPM)}

In the RPM, the sampling-based rational agent starts with a set of preferences for features typically observed in online services. For humans, these will be determined from previous experience. We adopt the approach of [32], who propose that preference formation can be "learned from experience without using option-specific subjective utilities". Under this assumption, humans make choices in the present by 
considering past experiences, but only a limited subset of them [33]. They can infer the relative desirability of an option by sampling the rate with which that option was chosen in a subset of past experiences, as well as sampling contextual information of the choice from memory (for example monetary values or brand representation associated with a purchasable object). Under a static subjective utility representation that ascribes some fixed value to an option, choosing an option that does not express maximal subjective utility would appear irrational. Considering the resource management involved in sampling preference information from experience, such a behavior may instead arise from the current set of sampled memories. It is important to stress again that the process of sampling is considered an analogy to recalling information from memory, and not a conscious act of the agent during the completion of a choice task.

When the agent observes features present in a given app offering, they need to sample their desirability from memory. Desirability of a feature is implemented as a Bernoulli trial, with the success probability indicating the rate with which options including said feature were chosen in the subset of past experiences. This rate also acts as an indicator of the intention to use an online service because of the presence of said feature. Vice versa, its inverse indicates the intention not to use an online service due to the present feature. Both rates lie between 0 and 1 and sum up to 1 . When the agent wants to infer the desirability of a service, they begin to draw samples from each feature's desirability distribution. A main component of our model is the formalization of when the agent should stop drawing additional samples. We expect this sampling length to be influenced by two factors: the expected value difference $\left(E V D_{t}\right)$ between the two hypotheses (a feature being desirable or being undesirable), and the volatility $\left(V_{t}\right)$ in estimation caused by the current sample. This reflects the intuition that the more desirable a feature is, its samples will more reliably produce successes and trigger an earlier termination from sampling. Vice versa, if a feature's desirability is less clear, samples are more likely to hover around a success parameter of $50 \%$, decreasing the probability of sampling termination. When more samples accumulate, however, the change in desirability caused by the current sample becomes relatively smaller, increasing the probability to terminate sampling as the number of samples rises. We implement this behavior by determining the probability to stop sampling with equation 2 :

$$
p(s t o p)=\left(\frac{1}{T} \sum E V D_{t}\right)-V_{t}
$$

At each sampling step, the agent computes the expected value difference $\left(E V D_{t}\right)$ between the two hypotheses of the Bernoulli trial and subtracts a volatility term $\left(V_{t}\right)$. The volatility refers to the parameter change of the success probability from the previous to the current sampling step [34].

For illustrative purposes, we present a numerical example. The agent represents their current knowledge over a feature's desirability as a beta distribution. When they start inferring whether a feature is desirable, they ascribe equal probability (0.5) to both hypotheses. Over their entire choice history, however, they may have chosen options including said feature $80 \%$ of the time. The agent produces a sample from their choice history with this success probability of 0.8 . After observing that the sample showed a success, they update their current estimation of the feature's success probability which results in a shift from 0.5 to 0.67 , with its inverse (the undesirability of the feature) shifting from 0.5 to 0.33 . At this point, they can determine the value difference between the two hypotheses (the feature being desirable or undesirable), which in this example is given by $0.67-0.33=0.34$. The change in success probability estimation from before the sample to after the sample is expressed in the volatility parameter. It is the absolute value difference between 0.5 and 0.67 , so 0.17 . Subtracting 0.17 from 0.34 gives the stopping probability at this sampling step of $0.34-0.17=0.17$. After one sample, the agent will terminate sampling with a $17 \%$ chance, while continuing and drawing the next sample with an $83 \%$ chance. At that point, depending on the outcome of the sample, the desirability estimate is updated, and the stopping rule is reevaluated.

The proposed probabilistic stopping rule lets the agent keep on sampling when evidence is inconclusive. The agent sequentially updates a beta distribution with a uniform prior (1, 1) to maintain an estimate of a feature's desirability. Once they terminate sampling, they adjust their desirability estimate to the value $\mathrm{q}$ as seen in equation 3 . Here, $\theta_{C D F}$ refers to the binomial cumulative density function following [29]. Adjusting $\mathrm{q}$ according to the cumulative density function reflects that the more successes the agent samples before termination, the more certain they become in their desirability estimate. $\mathrm{q}$ increases as a function of the number of samples $\mathrm{k}$ and the sampled feature desirability $p$.

$$
q=1-\theta_{C D F}\left(\left\lfloor\frac{k}{2}\right\rfloor, p, k\right)
$$

Integrating the final feature desirabilities q via Bayes' rule then results in the posterior desirability of the service. Following equation 1 , desirabilities for all features present in a stimulus are multiplied in the nominator, and divided by their opposites in the denominator. The proposed behavior of the sampling-based agent allows for some assumptions about their behavior. Implementing the aforementioned 
probabilistic stopping rule will let the agent stop the sampling process after just one draw, as proposed to be plausible by [29]. However, due to the randomness of the sampling process, sampling lengths can range from one or just a few more samples (single-digit) to much longer sampling sequences, especially when the success probability approaches 0.5 . When the agent draws only one sample and stops, they approximate probability matching [35]: the tendency to only choose the favorable option in proportion with its relative success probability. Drawing more samples will increase the rate with which the best option is picked, until at an unlimited number of samples, the agent deterministically chooses the best option. Sampling from an individual participant's feature preferences and integrating them via Bayes' rule, the RPM returns a single probability of choosing to install an app. Appendix A displays the RPM in pseudo code.

\subsubsection{Bayesian model (detBayes)}

We propose a "regular" Bayesian agent as the first alternative model. The agent works on a solely computational level and therefore does not sample from preference distributions. Instead, it integrates a participant's stated feature preferences directly via Bayes' rule. It chooses an option deterministically once that option's posterior probability is above $50 \%$. It therefore employs equation 1 as its decision rule. In addition, it represents a special case of the RPM in which the agent draws an infinite number of samples. Previous research underlines the assumption that the Bayesian agent will not adequately describe human online choice behavior, and efforts have been made to show the superiority of other mechanisms from a theoretical perspective [14]. However, to our knowledge a fully Bayesian model has not been formally implemented and evaluated empirically in this context. The Bayesian model returns a single, binary response to install or not install an app, based on whichever hypothesis has higher posterior probability. It is important to note that we do not assume the agent to choose that option with probability equal to the posterior, but deterministically. Interpreting the posterior probability of an option as the probability with which the agent should choose it would transfer the Bayesian model into a probability matching model [35].

\subsubsection{Take-the-best heuristic $(\operatorname{detTTB})$}

The second alternative model under investigation employs the take-the-best heuristic as a decision rule [36], [37]. It differs computationally from the previous two models in that its decision making is based on one reason, or one app feature in our example, rather than on the integration of multiple available reasons. The take-the-best heuristic considers features of choice options in order of their validity. An agent basing their behavior on the heuristic will stop looking for features as soon as they find one that discriminates between the available options. They will then choose the option that provides a positive cue value - the one that includes the desirable feature or excludes the undesirable one. Consider the example of an agent looking for a certain feature in a smartphone app. The take-the-best heuristic behaves deterministically in that a given set of preferences and available features will always lead to the same first discriminative feature, and that feature will always direct the decision. Therefore, it returns a single, binary response to install or not install an app, based on the first valid feature encountered.

\subsubsection{Probabilistic take-the-best heuristic (probTTB)}

With the take-the-best heuristic following a different rationale than the previous two models, the RPM and the Bayesian model, it still shares a similarity with the latter: it behaves deterministically. To introduce a third, probabilistic alternative model, we adopt an alteration of the take-the-best heuristic implemented by [38]. Here, the order of feature consideration is not set by feature validity but features to consider are drawn from a probability distribution where a feature's probability of being sampled is equal to its relative validity. Appendix B displays the probabilistic take-the-best heuristic in pseudo code. Given its deterministic and probabilistic variants, we considered the take-the-best heuristic as a suitable alternative model to determine both the influence of singular versus multi-reason decision making and the contribution of probabilistic allowances simultaneously. As the stimuli introduced were fictional and novel to participants, however, we did not consider a recognition process usually preceding the take-the-best heuristic.

\subsection{Model evaluation}

When comparing cognitive models with respect to their ability to explain data, it is important to account for possible free parameters. One way to approach these adjustable parameters is to fit them to a data set at hand, but this approach comes with the caveat of overfitting [39], [40]. While a model with free parameters tends to fit better to any set of data the more free parameters it has, it does not necessarily perform well in predicting new data. There are several ways to handle this issue, for example by considering information criteria that adjust a model's fit based on the number of free parameters [41] or by using leave-one-out cross-validation [42]. The evaluation method we chose to employ in this article is the Bayes Factor. It is a ratio of marginal likelihoods between two competing models, resulting in a single value that tells us how 
likely the data are under one model compared to another one. The marginal likelihood weighs all predictions a model can make, that is, all possible combinations of values of all free parameters, by their prior probability. Another way of saying this is that instead of fitting parameter values to an existing data set, we average out all free parameters [43] and arrive at the average prediction a model makes without being fit to data. Equation 4 shows the ratio of the marginal likelihood of model 1 to model 2 as the Bayes Factor (BF):

$$
B F_{12}=\frac{p\left(D \mid M_{1}\right)}{p\left(D \mid M_{2}\right)}
$$

Marginalizing the free parameters in both probabilistic models provides one average prediction per participant and task of whether they would choose for or against installing the presented app. The likelihood of the observed amount of install decisions given this average prediction then follows a Poisson binomial distribution [44], as success probabilities are not necessarily identical between individuals. Because of the deterministic nature of the detBayes and detTTB models, they assign the entire probability mass of their predictions to one specific proportion of install choices, while the RPM and the probTTB model assign probability mass to many plausible proportions of install choices. This means that a deterministic model can only outperform a probabilistic model if its prediction exactly matches the empirically observed proportion as it assigns zero likelihood to all other outcomes. It also follows that a probabilistic model with a broader range of predictions is penalized for its lack of specificity. The analysis was conducted in RStudio 1.0.153 and R 3.4.1.

\section{Results}

\subsection{Model comparison}

Each model provided one success probability per participant and stimulus for choosing to install the app. Using the probability mass function of the Poisson binomial distribution, we derived the marginalized likelihood of the observed install proportion given each model. This posed a challenge for the deterministic models. Both assumed a certainty of $100 \%$ in their predictions, leading them to assign all available probability mass to exactly one outcome of install proportions. In four out of five cases, neither deterministic model exactly predicted the correct install proportion, leading to a likelihood of zero for the observed data. This issue was, however, not limited to the deterministic models. For the case of the first app in the second study, the probTTB model had its expected value located so far from the actual empirical observation that its likelihood was zero as well. Calculating a Bayes Factor when one of the involved models' likelihoods is zero might cause some objection, as division by zero is arithmetically undefined. Seeing that we used the Bayes Factor to compare the relative quality of models, however, we proceeded by following computation rules in $\mathrm{R}$ and treated the resulting Bayes Factor as infinite, and as evidence in favor of the model with likelihood above zero.

With regards to the interpretation of Bayes Factors, we adopted the conventional classification proposed by [45] and relabeled by [43]. Here, Bayes Factors of above 3 indicate moderate evidence for the first model, Bayes Factors above 10 indicate strong evidence for it, continuing until a Bayes Factor of above 100, which signals extreme evidence towards the first model. Results of the Bayes Factor comparisons between the probabilistic models RPM and probTTB as well as their interpretations are shown in Table 3.

Table 3.

Bayes Factors for the model comparison between RPM and probTTB across app stimuli. Each Bayes Factor uses the marginal likelihood of the RPM as the numerator.

\begin{tabular}{ccc}
\hline Stimulus & Bayes Factor & Support level \\
\hline Study 1, App 1 & 0.43 & No support in either direction \\
Study 1, App 2 & 354153.40 & Extreme support for RPM \\
Study 1, App 3 & 51809.12 & Extreme support for RPM \\
Study 2, App 1 & + Infinity & Extreme support for RPM \\
Study 2, App 2 & 1.87 & Anecdotal support for RPM \\
\hline
\end{tabular}

Comparisons involving a deterministic model show almost unanimous results, with one exception. The detTTB model for app 2 in the second study is the only deterministic model that succeeded in predicting the precise observed install proportion. For a graphical depiction of this, see Appendix C.5. Therefore, all remaining comparisons between the probabilistic and deterministic models result in relative support of the probabilistic model. For app 2 in the second study, comparing the RPM to the detTTB results in a Bayes Factor of 0.09 and comparing the probTTB to the detTTB results in one of 0.05 , indicating strong evidence towards detTTB in this instance.

\subsection{Feature preference distributions and install choices}

Tables 4 and 5 show descriptive data concerning the inferred feature preferences used to predict participant choice behavior. In study 1 , features involving monetary benefits 
(purchase price, booking alert, and discounts) showed the least amount of preference deviation in our sample of participants, with feature levels indicating a monetary benefit being collectively preferred. Meanwhile, features not associated with monetary benefits were ranked more diversely. In study 2 , features were not as easily separable into features with graspable monetary benefit and those without, but variation in preferences regarding permission requests (contact access and phone status access) resembled the variation found in permission request preferences of study 1 .

In study 1, out of 103 total participants 35, 50, and 32 participants respectively indicated install choices for the three app offerings shown. In study 2 with a total of 112 participants, 17 and 65 participants respectively indicated install choices for the two app offerings shown there. Appendix $\mathrm{C}$ shows these install choices in relation to graphical representations of the predicted install choices per model.

Table 4.

Average feature preference (standard deviation in parentheses) as inferred by the ranking procedure for study 1 (in percent).

\begin{tabular}{ccc}
\hline Feature & Reference level & Preference values \\
\hline Price & $0.00 €$ & $85 \%(17 \%)$ \\
Price alert & On & $81 \%(19 \%)$ \\
Evaluation & Customer rating & $52 \%(31 \%)$ \\
GPS & Access & $43 \%(36 \%)$ \\
Calendar & Access & $44 \%(37 \%)$ \\
Price deals & Yes & $90 \%(11 \%)$ \\
\hline
\end{tabular}

Table 5.

Average feature preference (standard deviation in parentheses) as inferred by the ranking procedure for study 2 (in percent).

\begin{tabular}{ccc}
\hline Feature & Reference level & Preference values \\
\hline Price & $0.00 €$ & $87 \%(10 \%)$ \\
User reviews & 4.5 stars & $83 \%(22 \%)$ \\
Encryption & Yes & $84 \%(15 \%)$ \\
Multi-platform & Yes & $80 \%(13 \%)$ \\
Contacts & Access & $28 \%(30 \%)$ \\
Phone status & Access & $23 \%(27 \%)$ \\
\hline
\end{tabular}

\section{Discussion}

We have presented a rational process model (RPM) that predicted participant choices in two online surveys about smartphone apps. We showed that the model performed significantly better than a Bayesian agent without sampling (detBayes), and significantly better than a heuristic alternative (detTTB) in most cases. Compared to the probabilistic version of the take-the-best heuristic (probTTB), Bayes Factors showed extreme support for the RPM in three cases, anecdotal support in one case, and no support to either model in one case. The discussion of these results will be structured as follows: we investigate model behavior and performance first. Then we emphasize the benefits of formal cognitive modeling for online privacy research, before describing limitations of the current studies.

\subsection{Conceptual and empirical model comparison}

Considering the computational and algorithmic levels of cognitive systems simultaneously [26] has helped Bayesian cognitive modeling overcome several challenges regarding psychological plausibility and mathematical tractability. Previous research has highlighted computational advances necessary to implement rational process models [29], [33] [34], as well as compared their predictions to empirical data [46], [47]. The present article has applied rational process modeling to privacy-related online choice behavior, an area of research in which human decision making has mostly been described as boundedly rational. The bounded part of this type of rationality refers to the description of humans doing the best they can, given the cognitive limitations they are bound to. Whether one chooses to follow this line of argument or the concept of global rationality, where locally irrational behavior is produced by sampling, might ultimately be a distinction without a difference when it comes to observable behavior. In both cases, humans produce varying, inconsequential, and seemingly irrational behavior. Aiming to reproduce this behavior, the RPM's sampling process relied on a probabilistic stopping rule that utilized expected value difference and parameter estimation volatility on a sampling step basis, producing four samples on average. Considering the low number of samples, the agent showed a tendency towards probability matching - that is, only choosing the favorable option proportionally to its relative success probability, adjusted for the number of samples drawn. We find both the sampling duration and tendency towards probability matching to be in line with previous research [29], [32]. It used individual feature preferences elicited through a rank ordering task. This stated preference elicitation revealed a comparatively higher precision in the preference judgment of features that enabled monetary benefits (see tables 4 and 5). Meanwhile, potentially privacy-invasive functionalities (specifically, GPS and calendar access permissions in study 1, and contact and phone status access in study 2) were ranked lower, but also showed a noticeably wider range of rankings. 
While the RPM and detBayes were chosen to represent rational accounts of decision making by utilizing all available information, detTTB and probTTB are more traditionally associated with the concept of bounded rationality. The takethe-best heuristic represents one-reason decision making [48], a measure that can be implemented to avoid cognitive limitations and adapt to surroundings in which one good reason can be enough of a "selling point" to base a decision on. Despite its different reasoning mechanism, the deterministic variant of the heuristic (detTTB) showed similar rates of correct classification compared to detBayes. It is easily apparent that both deterministic models were unlikely to be favored by the evaluation via Bayes Factor, as that would require them to precisely predict the proportion of install choices for a given app. Even though detTTB achieved just that for the second app of the second study, we attribute this result to coincidence rather than either of the deterministic models being an adequate model of the data generating process. Turning toward the comparison of the two probabilistic models, we observe extreme support for the RPM relative to the probTTB model for three out of five stimuli, with the two remaining ones showing either no support for either model or anecdotal support for the RPM. The reason for these two outcomes is unclear, but an important aspect of model comparison has to be kept in mind: the best model under consideration is not necessarily a good model, and support for one can only be interpreted relative to its alternative model. While the data offered more support for the RPM than the probTTB model, all models under consideration consistently overestimated the empirical install proportion except for the second app of study 2 .

We observed a noticeable characteristic of the probTTB model for specific cases. Depending on an individual's distribution of preferences and app features available, it was possible for the probTTB model to result in 100 percent of possible sequences in favor of one option. In approximately $11.59 \%$ of cases, the probTTB model predicted a 100 percent chance of selecting an option and was wrong for $37 \%$ of those. When this happened, a deviating choice from the model prediction could not be explained by probabilistic behavior. Even though the model could compensate for these cases empirically, conceptually their existence provides a challenge for the one-reason decision rule that does not exist for the RPM, as the integration of multiple sampled features results in a probability of choosing a specific option below 100 percent for even extreme preference combinations. One could argue that the probTTB model suffered a disadvantage in this comparison, as we assumed that it deterministically chose the option that provided a positive cue value and only showed probabilistic behavior in the sequence of features under consideration. This appears to be the established decision rule for both deterministic [49] and probabilistic [38] applications of the take-the best heuristic however. In summation, both probabilistic models empirically outperformed their deterministic counterparts, and the data show extreme support for the RPM over the probTTB model for the majority of app stimuli. However, all models under consideration tended to overestimate the proportion of install choices observed in the empirical sample.

\subsection{Integrating probabilistic cognitive modeling with online pri- vacy research}

The RPM approach has provided a way to predict the occurrence of seemingly irrational choice behavior as a result of its probabilistic components. But how does it differ from other lines of research assuming a rational trade-off between perceived risks and benefits in online privacy research? The privacy calculus approach [6], [9], [50] tends to employ statistical data analysis, specifically structural equation modeling, rather than cognitive modeling. To quote [51]: „Understanding the mind is what makes an explanatory cognitive model distinct from a statistical predictive model - our parameters often reflect hypothesized cognitive processes or representations (e.g. attention, memory capacity, decision thresholds, etc.) as opposed to the abstract predictive parameters of, say, weights in a regression model." By using data analysis to infer the statistical weight of a perceived benefit of an online service on the user decision, privacy calculus research leaves itself vulnerable to this issue - offering statistical prediction of behavior without a process-level explanation for it. For further discussion on the difference between statistical and cognitive modeling, see [52].

Research describing privacy-related choice behavior as boundedly rational tends to relate to prospect theory [13], claiming human cognition to be biased and their probability weighting to be distorted without offering a formal perspective of why and how that might be. While prospect theory is a prominent description of such phenomena, it makes no statement about the psychological mechanism involved in distorting the weights of formerly objective probabilities. Meanwhile, rational process modeling does offer a psychologically plausible explanation of these phenomena, as it has been employed to provide a computational explanation of the anchoring bias [53], as well as subjective probability distortion [54]. In cases where heuristic decision making has been proposed as an explanation for online choice behavior, it has, to our knowledge, not been implemented as a formal cognitive model. This results in a missing capability to provide precise, falsifiable predictions.

Demonstrating the insight that can be generated by formal modeling, this article has shown that neither the deterministic take-the-best heuristic nor a sampling-free Bayesian model are suitable predictors of online choice behavior. 
Implementing formal cognitive models that enable us to test and predict the development of psychological phenomena appears more sensible than merely describing them. Consequently, we want to emphasize that for online privacy research to make sense of user behavior, it is necessary to approach the topic from more than a descriptive account. For example, consideration of feature desirability distributions in tables 4 and 5 could inspire one to adopt an arguably simpler explanation of privacy disclosure behavior than any computational model presented here: that users desired features with monetary benefits more than they disliked features with privacy-invasive consequences, and that this constitutes a sufficient explanation of disclosure behavior. It is important to point out that the feature desirability distributions in tables 4 and 5 are ill-suited for the prediction of user behavior by themselves: they do not include any process-level specification of preference formation, memory capacity, or decision rule. We take the view that arguing against the complexity of formal computational models by relying on verbal-conceptual alternatives [15] falls flat, at the latest, when pressed to make precise quantitative predictions about observed user behavior. The extent of such predictions is therefore underspecified in a quantitative sense. This further highlights the necessity for formal cognitive models that are as explicit as possible about their process-level characteristics and subsequent predictions [19], [55].

\subsection{Limitations}

The present studies were conducted in the form of two online surveys. We replicated the results of the first study in a second one, generalizing the findings to a second category of smartphone apps and involving a new empirical sample. With online privacy research having previously focused on the difference between behavioral intentions and actual choice behavior [1], the online setting with hypothetical app adoption however represents a clear limitation. In fact, the hypothetical nature of the study scenario might explain why all models under consideration overestimated install proportions. The discrepancy here could stem from the fact that participants had no intrinsic demand for the apps shown to them. This is also reflected in generally low proportions of install choices. The introduction of an app to a major mobile ecosystem app store however would not have been controllable with respect to the amount of information presented on the stimuli. In a trade-off between the realism of the stimulus distribution and the controlled attention to selected features, we chose to limit the former in favor of the latter. Doing so resulted in the stated preference ranking method, which is at best a rough approximation of participants' preferences. They were assumed to sample preferences from their memory of past experiences, but controlling that process is only feasible if the researcher introduces naïve participants to new stimuli they had no former association with and controls preference formation. In a context like app adoption, where this is not feasible, preference inference through discrete choices can provide a solution to this problem. The resulting feature preferences are, however, influenced by the presented set of features and details of the task; thus, they are neither static nor transferable to other choice sets. It is worth noting though that these properties do reflect the proposed characteristics of preference formation by sampling from experience.

The lack of individual information provided by both probabilistic models reveals another limitation of the current study. If choice behavior is indeed well described by a Bernoulli process with choice probability $q$ as proposed by the RPM, or the sampling of feature sequences in the heuristic case, then individual data points are devoid of interpretability. [29] base their assumptions about human cognition on the fact that behavior appears to express optimality only on average, relying on sub-optimal sampling procedures that reach optimality in the long run. This leaves researchers with one of two choices when investigating human decision making: design an experiment in which each individual completes a large number of trials, or average across the empirical sample. We chose the latter solution because the stimuli presented in this article were difficult to plausibly showcase in an amount reaching higher double-digits. Studies in other areas of decision making and perception rely on stimuli that are comparatively easier to create and display in large numbers, but subjecting participants to such an amount of app alternatives did not appear to increase the realism of the task.

\section{Conclusion}

\subsection{Probabilistic decision making and privacy disclosure behav- ior}

We have established that choice behavior with regards to the adoption of smartphone apps is best described by the predictions of a RPM. Features in the presented stimuli that were linked to potential monetary benefit increased the probability of adoption strongly. Potentially privacy-invasive features decreased that probability but were comparatively outweighed by the beneficial features. A rational agent, following Bayes' rule as a principle of mathematical rationality, would choose the option that maximizes perceived utility deterministically. Prior research had indicated that privacy disclosure behavior was not well described by this type of Bayesian rationality, and we did find that a corresponding cognitive model did not receive support from the empirical sample. The sampling-based agent proposed here does not deterministically maximize the task-related utility. Instead, it 
samples from individual feature desirability distributions until a probabilistic stopping rule is triggered. At that point, it stochastically chooses the option with higher perceived desirability. The more samples the agent draws, the more likely they engage in utility maximization. If they stop after only one sample, they engage in probability matching. The stopping rule of how many samples to draw reflects the decision about the amount of cognitive resources attributed to the task. We found that in a comparison between the sampling-free Bayesian model detBayes, a one-reason-focused alternative implementing the take-the-best heuristic (detTTB, [49]), a probabilistic alternative of said heuristic (probTTB, [38]), and the sampling-based RPM, the latter outperformed its competing models. Additionally, we observed specific trials that showed participants violating the predictions of the probTTB model when its probability to choose one option reached 100 percent. These special cases are currently inconsistent with the assumptions of the model, requiring further investigation to determine if they constitute an empirical disadvantage of the probTTB model compared to the RPM.

Following from these results, we can draw some conclusions about privacy disclosure behavior. The expression of preferences in a given choice is variable due to the sampling process involved. Therefore, privacy disclosure research should not expect invariant behavior from users when investigating the rationality of their choices. Assuming that choices involving online services are usually made fairly quickly, users only use a handful of samples to arrive at such choices. That implies a high tendency towards probability matching, as can be observed in the discrepancy between the detBayes prediction (representing desirability maximization) and the empirical data. Consequently, it becomes clear why not specifically investigating the choice process via probabilistic cognitive modeling may leave the impression of irrational behavior. The issue is not that users do not consider the risks of potentially privacy-invasive features. They rather do not invest enough resources in the form of longer sampling sequences to act deterministically.

\subsection{Implications for empowering privacy-preserving behavior}

Authorities, employers, teachers, parents, and other stakeholders who want to improve the quality of the choice process of their target groups (citizens, employees, students, children) in online scenarios need intervention tools that improve decision making. A possible starting point for such interventions would be intervention design that creates the necessity for users to draw more samples. Making them literally "think twice", restating the request for a specific permission an additional time on mobile devices may already provide them with a decisive break to trigger further sampling. The concept of obstructive user interface design [56] is an example of what attempts to trigger resampling might look in practice. This, however, would only result in more privacy-preserving behavior (i.e., not choosing to use services with potentially privacy-invasive features) if a privacy-invasive feature was reducing the individual desirability of a service. If the presence of a privacy-invasive feature was in fact increasing the desirability of a service because a user had previously not discovered any problematic aspect of the feature, then resampling would increase the average probability of using the service. In that case, another intervention approach would be to rely on knowledge transfer. This approach does not fit as seamlessly into the models proposed here, as it relies on establishing new knowledge rather than sampling existing information. It could however easily be integrated by implementing choice modeling from description rather than experience [57].

\subsection{Future research}

While the example of obstructive user interfaces fits well into a computational perspective, it is a fair assumption that users would stop using the corresponding services altogether or quickly ignore interface annoyances. Research in security interventions already provides solutions to maintain user attention to warning signals [58], but an effort should still be made to integrate affective components into cognitive modeling. For example, [54] have shown that surprise serves as an influential factor for memory retrieval. Correspondingly, computational analysis of the conditions necessary to make annoying interventions be considered useful may provide further insight into user choice behavior. To first generalize the findings of this article however, we plan to replicate the model for additional categories of smartphone apps and other online services, from which we expect different feature desirabilities. We began our work in this article with smartphone apps because of the comparative simplicity of app store offerings. However, we neglected a design aspect of current mobile app stores: usually, potentially privacy-invasive features of apps are stashed away behind several screens of marketing information, and permission requests are usually not shown when the user decides to install the service, but when they first encounter the specific functionality inside the app. This may lead to privacy-invasive features not being considered at all at the moment of service adoption. We will use future work to investigate the impact this design choice has on the decision-making process of users. 


\section{Acknowledgements}

This work has been funded by the DFG as part of project A.2 within the RTG 2050 "Privacy and Trust for Mobile Users". We would like to sincerely thank Dr. Paul Gerber and Max Maass, M.Sc. for their valuable feedback on this article.

\section{References}

[1] P. A. Norberg, D. R. Horne, and D. A. Horne, "The Privacy Paradox: Personal information disclosure intentions versus behaviors," Journal of Consumer Affairs, vol. 41, no. 1, pp. 100-126, Jun. 2007.

[2] T. Dienlin and S. Trepte, "Is the privacy paradox a relic of the past? An in-depth analysis of privacy attitudes and privacy behaviors: The relation between privacy attitudes and privacy behaviors," European Journal of Social Psychology, vol. 45, no. 3, pp. 285297, Apr. 2015.

[3] A. Acquisti, "Nudging privacy: The behavioral economics of personal information," IEEE Security \& Privacy Magazine, vol. 7, no. 6, pp. 82-85, Nov. 2009.

[4] C. Lutz and P. Strathoff, "Privacy concerns and online behavior not so paradoxical after all? Viewing the Privacy Paradox through different theoretical lenses," SSRN Electronic Journal, 2014.

[5] C. P. Hoffmann, C. Lutz, and G. Ranzini, "Privacy cynicism: A new approach to the privacy paradox," Cyberpsychology: Journal of Psychosocial Research on Cyberspace, vol. 10, no. 4, 2016.

[6] F. Kehr, D. Wentzel, and T. Kowatsch, "Privacy Paradox revised: Pre-existing attitudes, psychological ownership, and actual disclosure," in Thirty Fifth International Conference on Information Systems, Auckland, 2014.

[7] H. Krasnova, E. Kolesnikova, and O. Günther, “'It won't happen to me!': self-disclosure in online social networks," presented at the 15th Americas Conference on Information Systems (AMCIS 2009), Atlanta, Georgia, 2009.

[8] H. Lee, H. Park, and J. Kim, "Why do people share their context information on Social Network Services? A qualitative study and an experimental study on users' behavior of balancing perceived benefit and risk," International Journal of Human-Computer Studies, vol. 71, no. 9, pp. 862-877, Sep. 2013.

[9] T. Dinev and P. Hart, "An extended privacy calculus model for E-Commerce transactions," Information Systems Research, vol. 17, no. 1, pp. 61-80, Mar. 2006.
[10] O. Morgenstern and J. Von Neumann, Theory of games and economic behavior. Princeton, N.J.: Princeton university press, 1953.

[11] S. Kokolakis, "Privacy attitudes and privacy behaviour: A review of current research on the privacy paradox phenomenon," Computers \& Security, vol. 64, pp. 122-134, Jan. 2017.

[12] H. A. Simon, "A behavioral model of rational choice," The Quarterly Journal of Economics, vol. 69, no. 1, pp. 99-118, 1955.

[13] D. Kahneman and A. Tversky, "Prospect Theory: An analysis of decision under risk," Econometrica, vol. 47, no. 2, p. 263, Mar. 1979.

[14] A. Acquisti, "Privacy in electronic commerce and the economics of immediate gratification," 2004, p. 21.

[15] R. Sun, "Introduction to computational cognitive modeling," in The Cambridge Handbook of Computational Psychology, R. Sun, Ed. Cambridge: Cambridge University Press, 2008, pp. 3-20.

[16] G. Murphy, "The contribution (and drawbacks) of models to the study of concepts," in Formal Approaches in Categorization, E. Pothos and A. Willis, Eds. Cambridge University Press, 2011, pp. 299-312.

[17] M. Peterson, An introduction to decision theory. New York: Cambridge University Press, 2009.

[18] A. Acquisti and J. Grossklags, "Privacy and rationality in individual decision making," IEEE Security and Privacy Magazine, vol. 3, no. 1, pp. 26-33, Jan. 2005.

[19] I. Farkaš, "Indispensability of computational modeling in cognitive science," Journal of Cognitive Science, vol. 13, no. 12, pp. 401-435, 2012.

[20] R. Sun, Ed., The Cambridge handbook of computational psychology. Cambridge ; New York: Cambridge University Press, 2008.

[21] S. Farrell and S. Lewandowsky, Computational Modeling of Cognition and Behavior, 1st ed. Cambridge University Press, 2018.

[22] D. M. Eddy, "Probabilistic reasoning in clinical medicine: Problems and opportunities," in Judgment under uncertainty, D. Kahneman, P. Slovic, and A. Tversky, Eds. Cambridge: Cambridge University Press, 1982, pp. 249-267.

[23] D. Marr, Vision: A computational investigation into the human representation and processing of visual information. Cambridge, Mass.: MIT Press, 1982.

[24] T. L. Griffiths, E. Vul, and A. N. Sanborn, "Bridging levels of analysis for probabilistic models of cognition," Current Directions in Psychological Science, vol. 21, no. 4, pp. 263-268, Aug. 2012.

[25] A. N. Sanborn, T. L. Griffiths, and D. J. Navarro, "Rational approximations to rational models: Alternative algorithms for category learning.," Psychological Review, vol. 117, no. 4, pp. 1144-1167, 2010.

[26] T. L. Griffiths, F. Lieder, and N. D. Goodman, "Rational use of cognitive resources: Levels of analysis between the computational and the algorithmic," 
Topics in Cognitive Science, vol. 7, no. 2, pp. $217-$ 229, Apr. 2015.

[27] R. Hertwig, G. Barron, E. U. Weber, and I. Erev, "Decisions from Experience and the effect of rare events in risky choice," Psychological Science, vol. 15, no. 8, pp. 534-539, Aug. 2004.

[28] A. N. Sanborn and N. Chater, "Bayesian Brains without Probabilities," Trends in Cognitive Sciences, vol. 20, no. 12, pp. 883-893, Dec. 2016.

[29] E. Vul, N. Goodman, T. L. Griffiths, and J. B. Tenenbaum, "One and done? Optimal decisions from very few samples," Cognitive Science, vol. 38, no. 4, pp. 599-637, May 2014.

[30] M. Taddicken, "The Privacy Paradox in the Social Web: The Impact of Privacy Concerns, Individual Characteristics, and the Perceived Social Relevance on Different Forms of Self-Disclosure," Journal of Computer-Mediated Communication, vol. 19, no. 2, pp. 248-273, Jan. 2014.

[31] Z. Tufekci, "Can You See Me Now? Audience and Disclosure Regulation in Online Social Network Sites," Bulletin of Science, Technology \& Society, vol. 28, no. 1, pp. 20-36, Dec. 2007.

[32] N. Srivastava and E. Vul, "Choosing fast and slow: explaining differences between hedonic and utilitarian choices.," in Proceedings of the 37th Annual Meeting of the Cognitive Science Society, 2015.

[33] N. Srivastava and P. Schrater, "Frugal preference formation," in Proceedings of the 36th Annual Meeting of the Cognitive Science Society, 2014.

[34] N. Srivastava, J. Müller-Trede, P. Schrater, and E. Vul, "Modeling sampling duration in decisions from experience," in Proceedings of the 38th Annual Meeting of the Cognitive Science Society, 2016.

[35] D. R. Shanks, R. J. Tunney, and J. D. McCarthy, “A re-examination of probability matching and rational choice," Journal of Behavioral Decision Making, vol. 15, no. 3, pp. 233-250, Jul. 2002.

[36] G. Gigerenzer, "Why heuristics work," Perspectives on Psychological Science, vol. 3, no. 1, pp. 20-29, Jan. 2008.

[37] A. Graefe and J. S. Armstrong, "Predicting elections from the most important issue: A test of the take-thebest heuristic," Journal of Behavioral Decision Making, vol. 25, no. 1, pp. 41-48, Jan. 2012.

[38] F. B. Bergert and R. M. Nosofsky, "A response-time approach to comparing generalized rational and takethe-best models of decision making.," Journal of Experimental Psychology: Learning, Memory, and Cognition, vol. 33, no. 1, pp. 107-129, 2007.

[39] E. Brandstätter, G. Gigerenzer, and R. Hertwig, "The priority heuristic: Making choices without tradeoffs.," Psychological Review, vol. 113, no. 2, pp. 409-432, 2006.
[40] S. Roberts and H. Pashler, "How persuasive is a good fit? A comment on theory testing," Psychol Rev, vol. 107, no. 2, pp. 358-367, Apr. 2000.

[41] A. Gelman, J. Hwang, and A. Vehtari, "Understanding predictive information criteria for Bayesian models," Statistics and Computing, vol. 24, no. 6, pp. 997-1016, Nov. 2014.

[42] A. Vehtari, A. Gelman, and J. Gabry, "Practical Bayesian model evaluation using leave-one-out crossvalidation and WAIC," Statistics and Computing, vol. 27, no. 5, pp. 1413-1432, Sep. 2017.

[43] M. D. Lee and E.-J. Wagenmakers, Bayesian cognitive modeling: a practical course. Cambridge; New York: Cambridge University Press, 2013.

[44] Y. Hong, "On computing the distribution function for the Poisson binomial distribution," Computational Statistics \& Data Analysis, vol. 59, pp. 41-51, Mar. 2013.

[45] H. Jeffreys, Theory of probability, 3rd ed. Oxford: Clarendon Press ; Oxford University Press, 1998.

[46] E. Bonawitz, S. Denison, A. Gopnik, and T. L. Griffiths, "Win-Stay, Lose-Sample: A simple sequential algorithm for approximating Bayesian inference," Cognitive Psychology, vol. 74, pp. 35-65, Nov. 2014.

[47] A. N. Sanborn, T. L. Griffiths, and R. M. Shiffrin, "Uncovering mental representations with Markov chain Monte Carlo," Cognitive Psychology, vol. 60, no. 2, pp. 63-106, Mar. 2010.

[48] G. Gigerenzer and P. M. Todd, Simple heuristics that make us smart, 1. issued as an Oxford Univ. Press paperback. Oxford: Oxford Univ. Press, 2001.

[49] G. Gigerenzer and W. Gaissmaier, "Heuristic Decision Making," Annual Review of Psychology, vol. 62, no. 1, pp. 451-482, Jan. 2011.

[50] F. Kehr, T. Kowatsch, D. Wentzel, and E. Fleisch, "Blissfully ignorant: the effects of general privacy concerns, general institutional trust, and affect in the privacy calculus: Privacy calculus: dispositions and affect," Information Systems Journal, vol. 25, no. 6, pp. 607-635, Nov. 2015.

[51] M. N. Jones, "Developing cognitive theory by mining large-scale naturalistic data," in Big data in cognitive science, 2016, pp. 1-12.

[52] D. Mirman, "Statistical models vs. cognitive models," Minding the Brain, 2012. .

[53] F. Lieder, T. L. Griffiths, Q. J. M. Huys, and N. D. Goodman, "The anchoring bias reflects rational use of cognitive resources," Psychonomic Bulletin \& Review, May 2017.

[54] N. Srivastava and E. Vul, "Rationalizing subjective probability distortions," in Proceedings of the 39th Annual Meeting of the Cognitive Science Society, 2017.

[55] T. Stafford, "What use are computational models of cognitive processes?," in Connectionist models of behaviour and cognition II: proceedings of the Eleventh 
Neural Computation and Psychology Workshop, University of Oxford, UK, 16-18 July 2008, J. Mayor, N. Ruh, and K. Plunkett, Eds. 2009, pp. 265-274.

[56] T. Storer et al., "Encouraging second thoughts: Obstructive user interfaces for raising security awareness," PST, pp. 366-368, 2013.

[57] I. Erev et al., "A choice prediction competition: Choices from experience and from description," Journal of Behavioral Decision Making, vol. 23, no. 1, pp. 15-47, Jan. 2010

[58] N. Kolb, S. Bartsch, M. Volkamer, and J. Vogt, "Capturing attention for warnings about insecure password fields - systematic development of a passive security intervention," in Human Aspects of Information Security, Privacy, and Trust, vol. 8533, T. Tryfonas and I. Askoxylakis, Eds. Springer International Publishing, 2014, pp. 172-182.

\section{Appendix}

\section{A. Pseudocode for the RPM}

\begin{tabular}{lc}
\hline Algorithm 1 Sampling process to recall preferences from memory in the \\
RPM. & \\
\hline $1:$ & For 1:N participants \\
2: & For 1:M service features \\
3: & Initialization of beta distribution with beta $(a=1, b=$ \\
& 1) \\
4: & Repeat \\
5: & Bernoulli trial with success probability equal to fea- \\
& ture preference mn \\
6: & Update beta distribution \\
7: & Computation of $V$ and $E V D$ at step $t$ \\
$8:$ & Bernoulli trial to terminate sampling process with \\
9: & success probability equal to the result of equation 3 \\
& Until Bernoulli trial to terminate sampling process \\
10: & returns a success \\
\hline
\end{tabular}

\section{B. Pseudocode for probTTB}

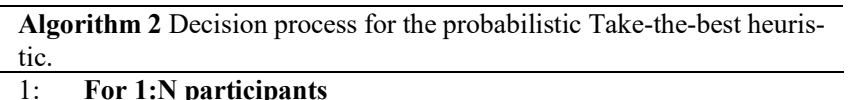

Determination of feature weights based on their preferences Repeat

Sampling of a feature with success probability proportional to its weight

Test for discriminability of sampled feature

Until feature discriminates between options

Choose option with higher preference regarding discriminating feature 


\section{Graphical representation of model predictions and empirically observed install choices}

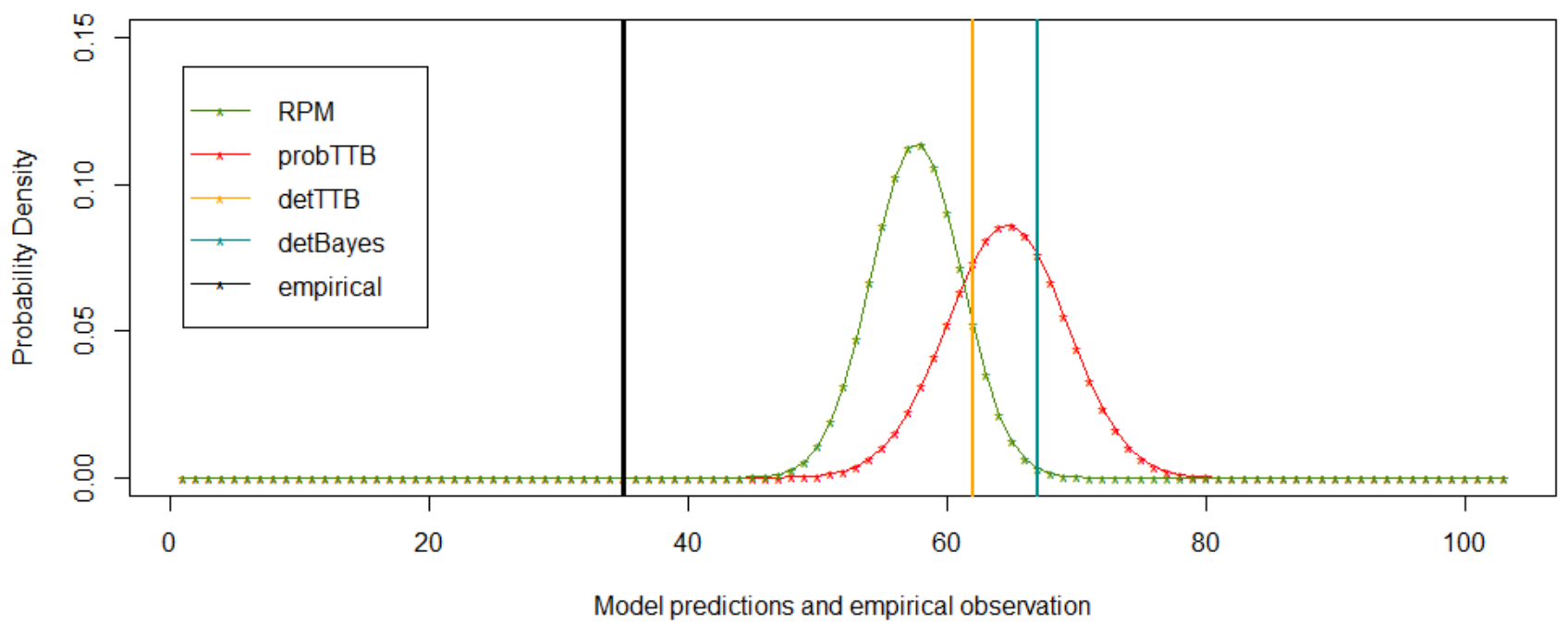

Appendix C.1 Model predictions and empirical observation of participant install choices for the first stimulus of study 1.

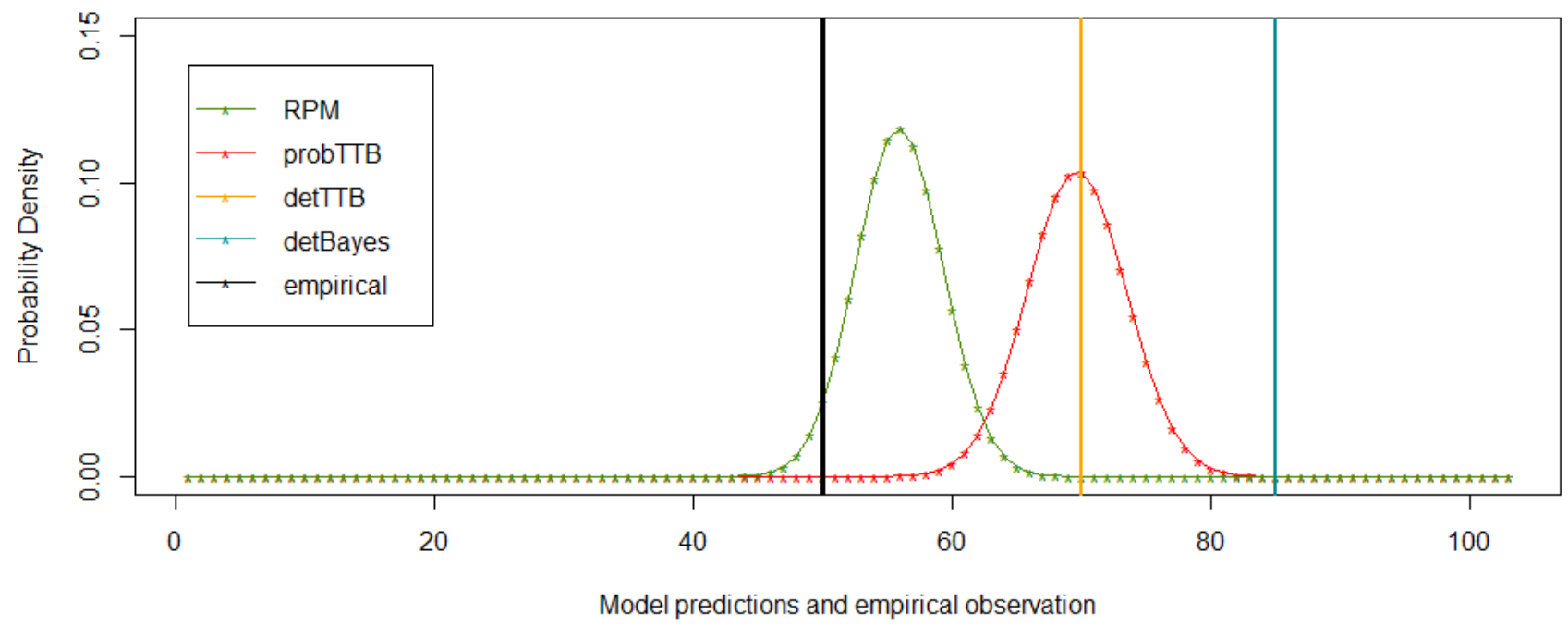

Appendix C.2 Model predictions and empirical observation of participant install choices for the second stimulus of study 1. 


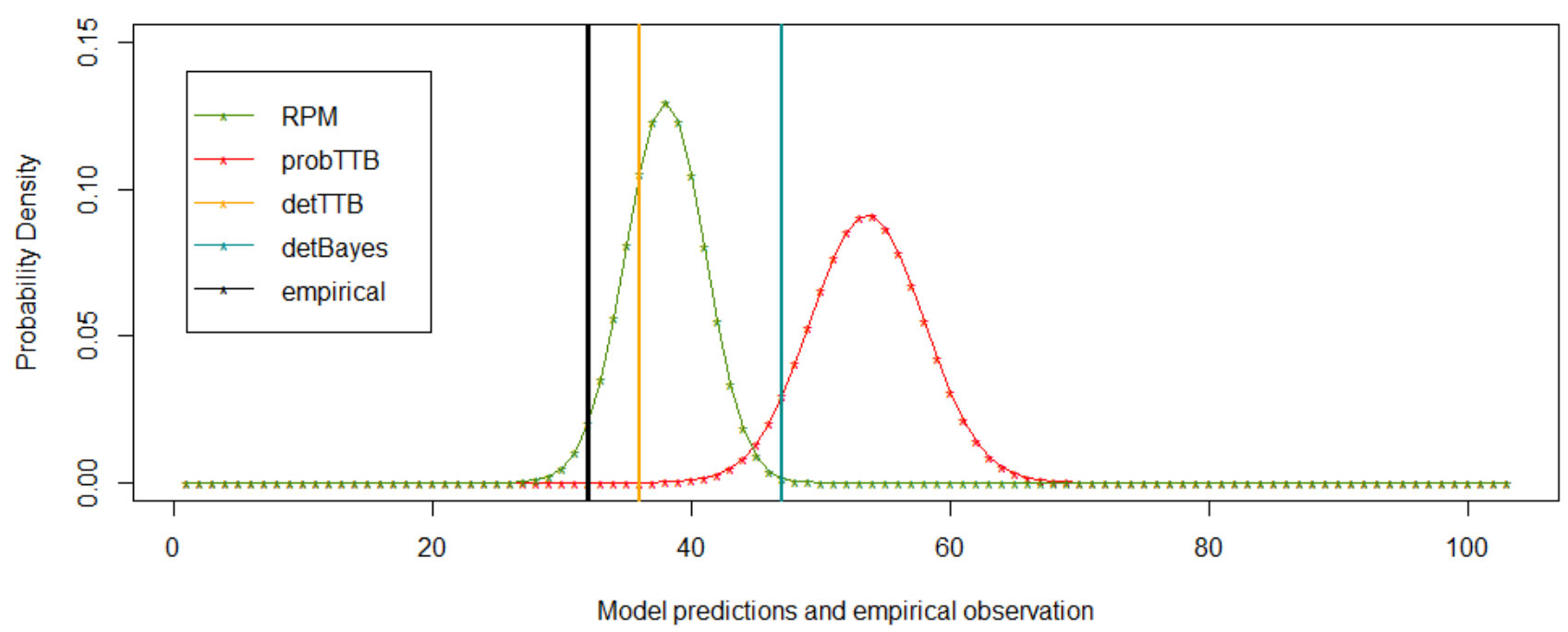

Appendix C.3 Model predictions and empirical observation of participant install choices for the third stimulus of study 1 .

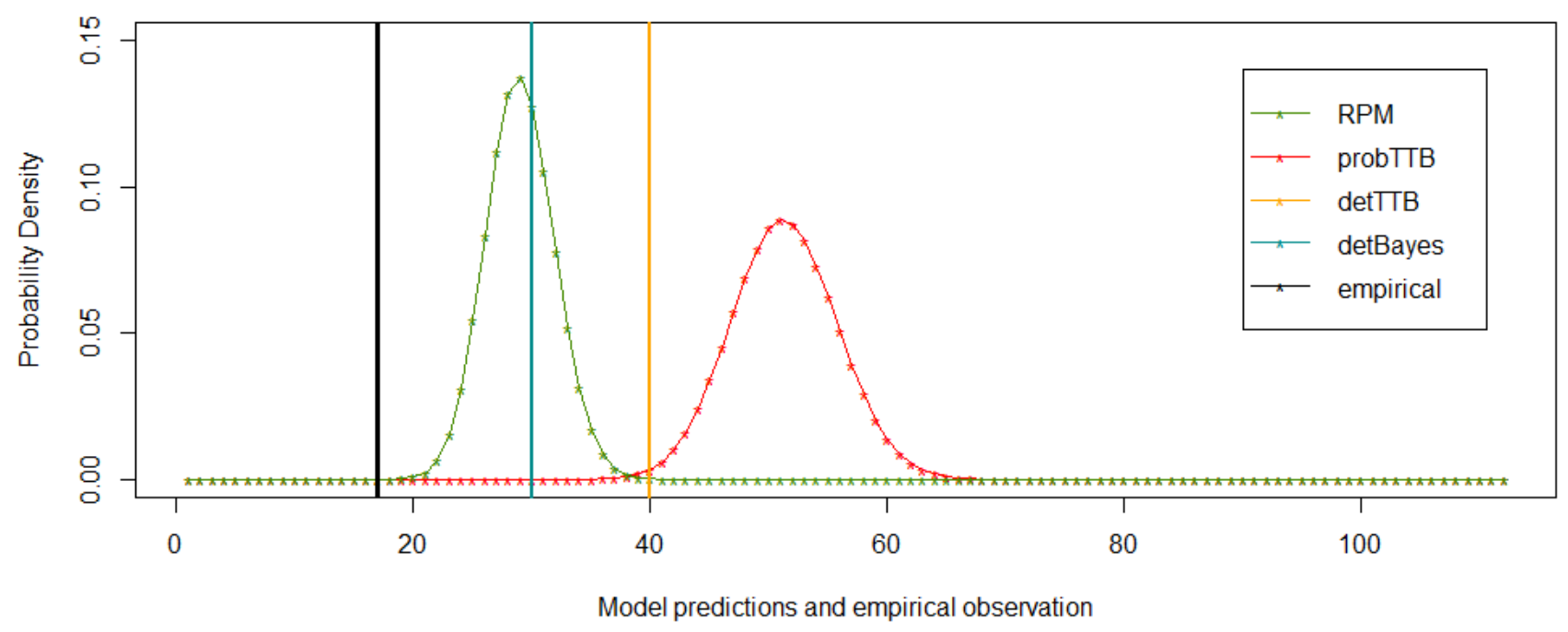

Appendix C.4 Model predictions and empirical observation of participant install choices for the first stimulus of study 2 . 


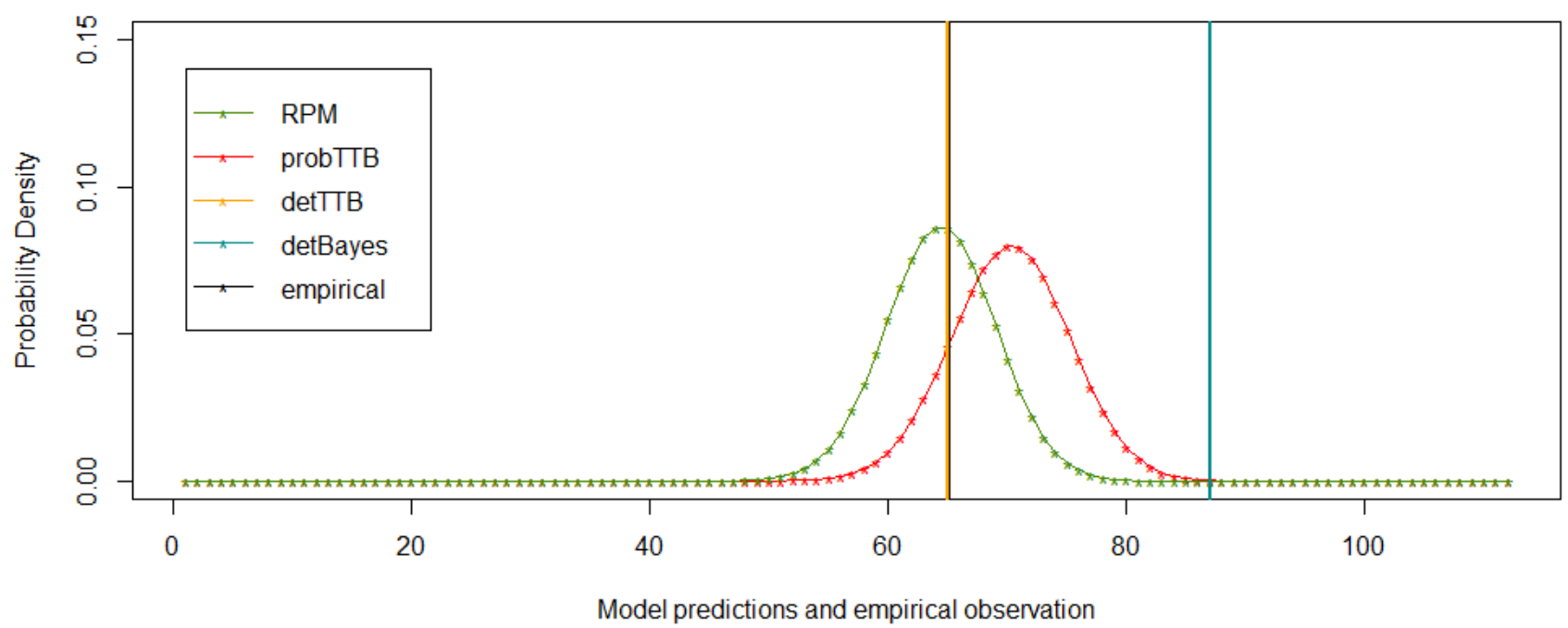

Appendix C.5 Model predictions and empirical observation of participant install choices for the second stimulus of study 2. 